\title{
Research into the Detonation of Non Ideal Versus Ideal Explosives
}

\author{
R.S. Miller and J.M. Goldwasser \\ Office of Naval Research, Mechanics and Energy Conversion Division, 800 North Quincy Street, \\ Arlington, VA 22217-5660, U.S.A.
}

The objectives are to determine, understand, and control the mechanisms and the rates of energy release in metallized explosive compositions during the detonation state and in the post-detonation regime. The goals of the initiative are to: (i) account for the total energy and the rates of energy release in the underwater detonation of a composite metallized explosive, (ii) establish and demonstrate concepts which can be employed to "dial" the near field pressure/time profile in order to maximize the desired energy coupling to the target, and (iii) develop a laboratory scale underwater detonation experiment, which will be able to accurately characterize the performance of novel metallized composite explosives. Research will be conducted to: (i) characterize the detonation both inside the explosive medium and in the water column at near-field, (ii) understand the parameters in the microstructural transport processes which govern the reactions of metal fuels with oxidizer molecules at temperature and pressure extremes, and the rates with which these processes take place, and (iii) identify the mechanisms by which energy is added, lost, or modified as the detonation wave and subsequent gas expansion propagate from the explosive into the water.

Our overall objectives are to determine and understand: (a) the mechanisms of chemical energy release and transport of mass, momentum, and energy in the detonation of heterogeneous metallized explosive compositions and (b) the resultant coupling of the released energy to the surrounding water medium. Underwater explosive formulations are non-ideal explosives. Non-ideal explosives are microscopically heterogeneous, detonable mixtures of oxidizer particles in fuel matrices that are separated by distances comparable to the multi-micron particle sizes of the oxidizer particles. Underwater explosives are inherently very different from ideal explosives used in air and surface applications. Ideal explosives are detonable, molecular mixtures of oxidizers and fuels, which are separated only by covalent or ionic bonds at Ångstrom distances, and release their energy very quickly compared to non-ideal explosives. In ideal explosives, the mixing and chemical reaction of shock decomposed oxidizer and fuel fragments occur quickly. The diffusion and the chemical reaction rates between reactive shocked oxidizer and fuel fragments are very fast, and the accompanying exothermic energy release rates to the reaction products occur over extremely short, Angstrom distances in contrast to the long, multi-micron distances for nonideal explosives. The fundamental differences are not only in the degree of heterogeneity, mixing distances, diffusion rates, reaction times and energy release rates but also in the environment in which they 
are employed. Large quantities of detonated ideal explosives work to rapidly accelerate and deform relatively small masses of metal in shaped charge and fragmenting warheads. In comparison, relatively small quantities of non-ideal explosives work to slowly accelerate relatively large masses of water. The inertial confinement of the environment of underwater explosives by the surrounding mass of water is, therefore, much greater than the inertial confinement of metal surrounding ideal explosives of shaped charges or fragmenting warheads. One of the consequences of the differences described above is in the failure diameter of composite explosives. Because of the heterogeneities of the explosive composition and relative slowness of reaction, the diameter of the explosive required to sustain a detonation is typically in the centimeter range as opposed to the millimeter range for ideal explosive compositions. The importance to science lies in understanding the microscopic kinetics of transport and chemical energy release processes of non-ideal detonations, and the time dependent coupling of these energy release processes to macroscopic hydrodynamic phenomena. As a result, the early time and near field chemistry and physical phenomena that govern the energy release mechanisms for these heterogeneous formulations are much slower than those of homogeneous explosives. However, they are much more complex, and have been very difficult to study and characterize. Of interest are the detonation wave and in the early postdetonation gas expansion. Our specific objectives are: (a) the characterization of the in-situ and near-field energy release profile of metallized composite explosives and the understanding of the energy losses and shock wave attenuation resulting from transition from the explosive medium through the interfacial layer into the water column, (b) the identification of the chemical reaction pathways; the micromechanical transport mechanisms responsible for mixing of metal/oxidizer fragments under shock loading; the characterization of the reaction kinetics as a function of temperature and pressure (up to $3000 \mathrm{~K}$ and 30 $\mathrm{GPa}$ ); introduction of pressure dependent chemical reaction parameters into metal combustion models, and (c) the establishment of a laboratory scale 1D experiment to characterize the underwater detonations of novel metallized composite explosives and the introduction of the time dependent energy release parameters into equation of state predictions. Some specific research issues are: (a) How accurately can the in-situ and near-field pressure/time histories, positions, velocities, and curvatures of the shock and expanding bubble fronts be determined for metallized explosives using advanced diagnostic techniques? (b) What are the energy addition, loss, and shock wave attenuation processes which affect the pressure/time profile as the detonation wave proceeds from the explosive into the water medium through the interfacial layer? (c) How much can one probe in real time the chemical reactions in single particle and microdetonics experiments? (d) Are the spectroscopic signals strong enough and relaxation fast enough to use vibrational spectroscopy to accurately follow the reaction temperature during the detonation and early post-detonation regimes? (e) What is the regression mechanism of the metal fuel particles under detonation conditions and how would changing the detonation products from solids to gases affect the energy release rates and coupling efficiency to the medium? (f) Can shock initiated solid state reactions be successfully used to modify the reaction temperature and detonation kinetics? (g) Can a 1D laboratory scale underwater detonation experiment be implemented to accurately characterize novel metallized composite explosives in terms of pressure, temperature, velocity and shape of the shock wave, and rate of expansion of the compressed gas bubble in order to generate equations of state which account for the timedependent events which will be valid to predict large scale characteristics and performances? 